\title{
The Asymptotic Properties of Turbulent Solutions to the Navier-Stokes Equations
}

\author{
Zdeněk Skalák \\ Faculty of Civil Engineering, Czech Technical University in Prague, Thákurova 7, 16629 Prague, Czech Republic \\ Corresponding author: skalak@mat.fsv.cvut.cz
}

\begin{abstract}
In this paper we study the large time behavior of solutions to the Navier-Stokes equations. We present a brief survey of results concerning energy decay, and discuss a related phenomenon of the large time energy concentration in the frequency space occurring in any turbulent solution. This leads us to the study of solutions in the Besov spaces and to proof that if we choose a suitable initial condition then in some Besov spaces the energy of the associated solution does not decrease asymptotically to zero.
\end{abstract}

Keywords: Navier-Stokes equations, Besov spaces.

\section{Introduction}

We consider the Navier-Stokes equations for a viscous incompressible fluid which fills the whole threedimensional space $\mathbf{R}^{3}$ with the absence of external forces:

$$
\begin{aligned}
\partial_{t} u+\nabla \cdot(u \otimes u) & =\Delta u-\nabla p, \\
\nabla \cdot u & =0, \\
u(x, 0) & =u_{0}(x) .
\end{aligned}
$$

Here $u: \mathbf{R}^{3} \times[0, \infty) \rightarrow \mathbf{R}^{3}$ denotes the unknown velocity field and $p: \mathbf{R}^{3} \times[0, \infty) \rightarrow \mathbf{R}$ is the unknown pressure. $u_{0}=u_{0}(x)=\left(u_{01}(x), u_{02}(x), u_{03}(x)\right)$ is a given initial velocity.

The mathematical theory of the Navier-Stokes equations has been developed since the pioneering work by Leray ([7]). Plenty of papers and books can now be found in the literature concerning various aspects of the theory, among them the famous problem (still unresolved) whether a solution of the Navier-Stokes equations with smooth data remains regular for all times or can develop a blow-up in a finite time. In this paper we are interested in the large time behavior of the solutions and we start with the following basic question: does the kinetic energy of the solutions decrease to zero as time $t$ goes to infinity? This question was first raised by Leray in [7] in 1934 and the intuitive answer is positive, since we consider no external forces here. Indeed, the answer "yes" turns out to be correct, but many years passed between the formulation of the question and its partial solution by Kato, in [6]. Having solved the basic problem, we can now investigate more detailed aspects of energy decay. In the second section, we will discuss the rate of energy decay and we will present a short survey of the results.
The third section will be devoted to the phenomenon of large time energy concentration in solutions. It turns out that in every (turbulent) solution the energy concentrates for large times in frequencies forming an annulus or a ball in the frequency space. This phenomenon seems to be connected with the rate of energy decay discussed in the second section, and we will present several results concerning the existence and the rate of the energy concentration

The main results of this paper are presented in the fourth section, where we will turn our attention to the existence of solutions in Besov spaces. These spaces are defined by the use of the Fourier transform, and enable a study of the location of the energy in the whole spectrum of frequencies. This seems to be a suitable way to study the problems presented in the third section. We improve a result presented by Miyakawa in [8], and show here that there exist some Besov spaces in which some solutions do not decrease asymptotically to zero, unlike the decrease to zero in the energy norm mentioned above.

For the purposes of clarity, all the notation used in this paper, and also definitions of some basic mathematical terms, can be found in the Appendix.

\section{Rate of energy decay}

As was mentioned in the Introduction, the energy of every turbulent solution $u$ decreases asymptotically to zero, i.e. $\lim _{t \rightarrow \infty}\|u(t)\|_{2}=0$. (A precise simple proof can be found in [20]).

A further logical step is to study the rate of energy decay, and to present some classes of initial conditions providing various rates of decay. Many results concerning this problem were proved by Schonbek (see, for example [11], [12] and [13]). We mention here 
as an example a result proved in [12]: If the initial condition $u_{0}$ belongs to the space $L^{1} \cap L_{\sigma}^{2}$, then there exists a global weak solution of (1)-(3) and $c>0$ such that

$$
\|u(t)\|_{2} \leq c(t+1)^{-1 / 4}
$$

for every $t \geq 0$. A key paper was published by Wiegner in [20]. He showed that, roughly speaking, most of the solutions of the Navier-Stokes equations decrease at the same rate as the solutions of the so called Stokes equations (the Navier-Stokes equations deprived of the nonlinear term) with the same initial conditions. More precisely, a turbulent solution with the initial condition $u_{0}$ decreases at the rate $(1+t)^{-\alpha}$ for some $\alpha \in(0,5 / 4]$ if also $e^{t \Delta} u_{0}$ decreases at the same rate. Solutions with an even higher rate of decay were studied by Miyakawa and Schonbek in [9]. They proved the following result:

Theorem 1. Let $u_{0} \in L_{\sigma}^{2}$ and $\int\left|u_{0}(x)\right|(1+|x|) d x<$ $\infty$. Let $u$ be a turbulent solution to the NSE with the initial condition $u_{0}$ such that $\|u(t)\|_{2} \leq C(1+t)^{-5 / 4}$. We set

$$
b_{h, k}=\int x_{h} u_{0 k}(x) d x
$$

and

$$
\Lambda_{h, k}=\int_{0}^{\infty} \int\left(u_{h} u_{k}\right)(x, t) d x d t \quad(h, k=1,2,3) .
$$

Then if $\left(b_{h, k}\right) \equiv 0$ and if there exists $C \in \mathbf{R}$ such that $\Lambda_{h, k}=C \delta_{h, k}$, then

$$
\lim _{t \rightarrow \infty} t^{5 / 4}\|u(t)\|_{2}=0 .
$$

Conversely, if $\left(b_{h, k}\right) \neq 0$ or $\left(\Lambda_{h, k}\right)$ is not scalar, then

$$
\liminf _{t \rightarrow \infty} t^{5 / 4}\|u(t)\|_{2}>0 .
$$

Theorem 1 presents conditions under which a solution decreases at a higher rate than $(1+t)^{-5 / 4}$. However, while it is simple to fulfill the condition $\left(b_{h, k}\right) \equiv 0$ just by a suitable choice of the initial condition, it is difficult to observe the condition $\Lambda_{h, k}=C \delta_{h, k}$, since it includes the solution itself. Thus, Theorem 1 neither ensures the existence of solutions decreasing at a rate quicker than $(1+t)^{-5 / 4}$ nor gives a method for possibly constructing such solutions. This problem is solved by Brandolese in [1]. He used the following concept of a symmetric solution:

Definition 1. A vector field $u_{0}=\left(u_{01}, u_{02}, u_{03}\right)$ from $\mathbf{R}^{3}$ to $\mathbf{R}^{3}$ is said to be symmetric if the following conditions are satisfied for all $j, k=1,2,3$.

1. $u_{0 j}$ is odd with respect to $x_{j}$ and even with respect to $x_{k}, j \neq k$.
2. $u_{01}(x)=u_{02}(\sigma x)=u_{03}\left(\sigma^{2} x\right)$, where $\sigma$ is the cycle $\sigma\left(x_{1}, x_{2}, x_{3}\right)=\left(x_{3}, x_{1}, x_{2}\right)$.

A simple example of a symmetric and solenoidal vector field is given by

$$
u_{0}\left(x_{1}, x_{2}, x_{3}\right)=\left(\begin{array}{c}
x_{1}\left(x_{3}^{2}-x_{2}^{2}\right) e^{-|x|^{2}} \\
x_{2}\left(x_{1}^{2}-x_{3}^{2}\right) e^{-|x|^{2}} \\
x_{3}\left(x_{2}^{2}-x_{1}^{2}\right) e^{-|x|^{2}}
\end{array}\right) .
$$

Brandolese proved in [1] that if the initial condition $u_{0}$ is a solenoidal symmetric vector field then there exists a solution which is symmetric for every time $t \geq 0$. Now the existence of a solution decreasing at the rate $o\left((1+t)^{-5 / 4}\right), t \rightarrow \infty$ is ensured: it suffices to take the initial condition (4). Indeed, the associated solution $u=u(t)$ is then symmetric for every time $t \geq 0$ and it is also possible to verify the veracity of the condition $\Lambda_{h, k}=C \delta_{h, k}$. Moreover, the initial condition (4) also satisfies $\left(b_{h, k}\right) \equiv 0$ and the above conclusion follows from Theorem 1 .

Using symmetric initial conditions, Brandolese obtained solutions decreasing at the rate $(1+t)^{-9 / 4}$.

Obtaining solutions which decrease at an even higher rate seems to be difficult, with only one exception: The existence of exponentially decreasing solutions is very well known, as was described in [10] and [17]. These solutions are very rare since it is possible to prove that their initial conditions lie only on a thin manifold in the phase space. The direct construction of exponentially decreasing solutions in three dimensional spaces is still an open problem.

\section{Concentration of energy}

In this section, we present several results concerning the large time energy concentration which occurs in every (turbulent) solution: the energy of the solution concentrates in frequencies localized in an annulus (for the case of solutions decreasing exponentially) or a ball (for the case of solutions NOT decreasing exponentially) in the frequency space. The diameter of the annulus determines the rate of the exponential decay of the solution, and it can be arbitrarily narrow. The ball is centered in the origin of the coordinates, and can have an arbitrarily small diameter (for the results presented in this section, see [15], [16], [17] and $[18])$. The following theorem and the ensuing remarks provide precise information.

Theorem 2. Let $u$ be a nonzero turbulent solution of (1)-(3). Then there exists $a \in[0, \infty)$ such that

$$
\lim _{t \rightarrow \infty} \frac{\left\|\left(E_{a+\varepsilon}-E_{a-\varepsilon}\right) u(t)\right\|_{2}}{\|u(t)\|_{2}}=1
$$

for every $\varepsilon>0$, where we put $E_{a-\varepsilon}=0$ if $a-\varepsilon<$ 0 . The number a can be explicitly computed as $a=$ $\lim _{t \rightarrow \infty}\left\|A^{1 / 2} u(t)\right\|_{2}^{2} /\|u(t)\|_{2}^{2}$. 
Further,

$$
a=\sup \left\{\lambda \geq 0 ; \lim _{t \rightarrow \infty}\|u(t)\|_{2} e^{\lambda t}=0\right\},
$$

which implies that the energy of $u$ decreases exponentially for $t \rightarrow \infty$ if and only if $a>0$.

Finally, if $a>0$ and $\varepsilon>0$, then

$$
\lim _{t \rightarrow \infty} e^{(a-\varepsilon) t}\|u(t)\|_{2}=0
$$

and

$$
\lim _{t \rightarrow \infty} e^{(a+\varepsilon) t}\|u(t)\|_{2}=\infty .
$$

Remark 1. It is possible to show (see [5]) that for every $\lambda>0$

$$
F\left(E_{\lambda} u(t)\right)(\xi)=\chi_{B_{\sqrt{\lambda}}(0)} F(u(t))(\xi),
$$

where $F$ denotes the Fourier transform and $\chi_{B_{\sqrt{\lambda}}(0)}$ is the characteristic function of $B_{\sqrt{\lambda}}(0)=\{x \in$ $\left.\mathbf{R}^{3} ;\|x\| \leq \sqrt{\lambda}\right\}$. Consequently, the equality (5) can be written in the form

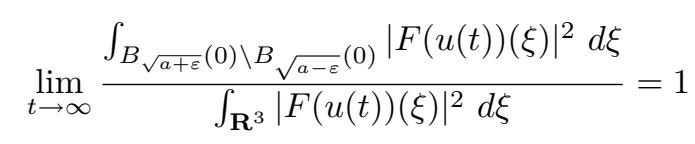

if $a>0$ and $\varepsilon \in(0, a)$ and

$$
\lim _{t \rightarrow \infty} \frac{\int_{B_{\sqrt{\varepsilon}}(0)}|F(u(t))(\xi)|^{2} d \xi}{\int_{\mathbf{R}^{3}}|F(u(t))(\xi)|^{2} d \xi}=1
$$

if $a=0$ and $\varepsilon>0$.

The results from Theorem 2 and Remark 1 can be interpreted in a way that in every turbulent solution the frequencies outside the annulus or the ball disappear asymptotically. This result can be further strengthened in the following way:

Theorem 3. Let $\alpha \geq 0$. Then

$$
\lim _{t \rightarrow \infty} \frac{\int_{K_{a, \varepsilon}^{C}}|\xi|^{4 \alpha}|F(u(t))(\xi)|^{2} d \xi}{\int_{\mathbf{R}^{3}}|F(u(t))(\xi)|^{2} d \xi}=0,
$$

where $K_{a, \varepsilon}^{C}=\mathbf{R}^{3} \backslash K_{a, \varepsilon}, K_{a, \varepsilon}=B_{\sqrt{a+\varepsilon}}(0) \backslash B_{\sqrt{a-\varepsilon}}(0)$ if $a>0$ and $K_{a, \varepsilon}=B_{\sqrt{\varepsilon}}(0)$ if $a=0$.

Up to now we have discussed the phenomenon of the large time energy concentration in the frequency space which occurs in any turbulent solution. In Theorem 4, we will present an example of a concrete class of initial conditions such that if $u_{0}$ belongs to this class and $u$ is a turbulent solution with $u(0)=u_{0}$, then the energy of the solution concentrates asymptotically in frequencies from an arbitrarily small ball in the frequency space centered in the origin of the coordinates. We also present two estimates of the rate of energy concentration (see [18]).

For a description of the class mentioned in the previous paragraph we need the following definition.
Definition 2. Let $\alpha, \delta>0$, and $m$ be a real number. We define

$$
K_{m, \alpha}^{\delta}=\left\{v \in L_{\sigma}^{2} ;|F(v)(\xi)| \geq \alpha|\xi|^{m}, \forall|\xi| \leq \delta\right\} .
$$

Theorem 4. Let $\alpha, \delta>0, m>-3 / 2, p \in[1,2]$ and

$$
\frac{3}{p}-\frac{3}{2} \leq m+3 / 2<\min \left(\frac{6}{p}-\frac{5}{2}, \frac{5}{2}\right) .
$$

Let further

$$
u_{0} \in L_{\sigma}^{2} \cap K_{m, \alpha}^{\delta} \cap L^{p}
$$

and $u$ be a turbulent solution of (1)-(3) with the initial condition $u_{0}$.

If $q \geq 1 / 2$, then there exists $c>0$ dependent only on $\left\|u_{0}\right\|_{2},\left\|u_{0}\right\|_{p}, \delta, m, \alpha$ and $q$ such that

$$
1-\frac{\left\|E_{\lambda} u(t)\right\|_{2}}{\|u(t)\|_{2}} \leq \frac{c}{\lambda} t^{-1+(m-3 / p+3) /(2 q)}
$$

for every $\lambda>0$ and every $t \geq 1$.

Let $\lambda_{0}>0$. Then there exists $c>0$ dependent only on $\left\|u_{0}\right\|_{2},\left\|u_{0}\right\|_{p}, \delta, m, \alpha$ and $\lambda_{0}$ such that

$$
1-\frac{\left\|E_{\lambda} u(t)\right\|_{2}}{\|u(t)\|_{2}} \leq \frac{c}{\lambda^{2}} t^{-3 / p-1}
$$

for all $\lambda \geq \lambda_{0}$ and $t \geq 1$.

Inequalities (6) and (7) provide information about the concentration of the energy in low frequencies and about the rate of this concentration.

Since the energy of the solutions described in Theorem 5 concentrates at low frequencies, the number $a$ from Theorem 2 is equal to zero and the energy of these solutions does not decrease exponentially.

Let us mention here one open problem: to find a turbulent solution $u$ with an initial condition $u_{0}$ such that the number $a$ from Theorem 2 is positive. In other words, to find a solution with the energy decreasing at the exponential rate $e^{-a t}, t \rightarrow \infty$ and concentrating in frequencies from an arbitrarily narrow annulus with the middle diameter $a>0$.

\section{Solutions in Besov spaces}

We start this section with a definition of homogeneous Besov spaces (see also [2]).

Let $C$ be the annulus $\left\{\xi \in \mathbf{R}^{3} ; 3 / 4 \leq|\xi| \leq 8 / 3\right\}$. There exist the smooth radial function $\chi$ and $\varphi$ with the support $B(0,4 / 3)$ and $C$, resp., with values in $[0,1]$, and such that

$$
\begin{aligned}
\chi(\xi)+ & \sum_{j \geq 0} \varphi\left(2^{-j} \xi\right)=1, \forall \xi \in \mathbf{R}^{3} \\
\sum_{j \in Z} \varphi\left(2^{-j} \xi\right) & =1, \forall \xi \in \mathbf{R}^{3} \backslash\{0\},
\end{aligned}
$$




$$
\begin{gathered}
\operatorname{Supp} \varphi\left(2^{-j} \cdot\right) \cap \operatorname{Supp} \varphi\left(2^{-j^{\prime}} .\right)=\emptyset \text { if }\left|j-j^{\prime}\right| \geq 2, \\
\operatorname{Supp} \chi \cap \operatorname{Supp} \varphi\left(2^{-j} \cdot\right)=\emptyset \text { if } j \geq 1 .
\end{gathered}
$$

Let $h=F^{-1} \varphi$. If $u \in S^{\prime}$ (the space of tempered distributions), then the homogeneous dyadic blocks are defined for $j \in Z$ as

$$
\Delta_{j} u=2^{3 j} \int_{\mathbf{R}^{3}} h\left(2^{j} y\right) u(x-y) d y .
$$

The space of the homogeneous distributions $S_{h}^{\prime}$ is defined in the following way: $u \in S^{\prime}$ belongs to $S_{h}^{\prime}$ if and only if $u=\sum_{j \in Z} \Delta_{j} u$.

We can now define the homogeneous Besov space $B_{p, \infty}^{s}, s \in \mathbf{R}, p \in[1, \infty]$. This space consists of those distributions from $S_{h}^{\prime}$ such that

$$
\|u\|_{B_{p, \infty}^{s}}=\sup _{j \in Z} 2^{j s}\left\|\Delta_{j} u\right\|_{p}<\infty .
$$

Suppose now that $u$ is a turbulent solution of (1) (3) with an initial condition $u_{0}$. Then (see [19])

$$
u(t)=e^{t \Delta} u_{0}+\int_{0}^{t} e^{\Delta(t-s)} P_{\sigma} \nabla(u \otimes u(s)) d s .
$$

If we denote the integral from the previous equality as $w(t)$ and use the fact that the operator $P_{\sigma} \nabla$ is homogeneous of degree 1 , we can derive

$$
\begin{array}{r}
\left\|\Delta_{j} w(t)\right\|_{1} \\
\quad \leq \int_{0}^{t} C e^{-c(t-s) 2^{2 j}} 2^{j}\left\|\Delta_{j}(u \otimes u(s))\right\|_{1} d s .
\end{array}
$$

So, we have for every $t>0$

$$
\begin{gathered}
\|w(t)\|_{B_{1, \infty}^{-1}}=\sup _{j \in Z} 2^{-j}\left\|\Delta_{j} w(t)\right\|_{1} \\
\leq C \sup _{j \in Z} \int_{0}^{t} e^{-c(t-s) 2^{2 j}}\left\|\Delta_{j}(u \otimes u(s))\right\|_{1} d s \\
\leq C \sup _{j \in Z} \int_{0}^{t} e^{-c(t-s) 2^{2 j}}\|u(s)\|_{2}^{2} d s \\
\leq C \int_{0}^{t}\|u(s)\|_{2}^{2} d s<\infty .
\end{gathered}
$$

It follows that $w(t) \in B_{1, \infty}^{-1}$ and so $w(t) \in B_{2, \infty}^{-5 / 2}$, since $B_{1, \infty}^{-1}$ is continuously embedded into $B_{2, \infty}^{-5 / 2}$ (as follows from the Bernstein inequalities, see [3]). Thus, if the initial condition $u_{0}$ is from the space $B_{2, \infty}^{-5 / 2}$, then $e^{\Delta t} u_{0}$ is also from the same space. This means that $u(t) \in B_{2, \infty}^{-5 / 2}$ for every $t>0,\left\|e^{t \Delta} u_{0}\right\|_{2}$ decreases at the rate $(1+t)^{-5 / 4}$ (see [3]) and using the result from [20] mentioned in the second section we also have $\|u(t)\|_{2} \leq c_{2}(1+t)^{-5 / 4}$ for every $t \geq 0$.

Suppose now that the initial condition was chosen in such a way that

$$
c_{1}(1+t)^{-5 / 4} \leq\|u(t)\|_{2}
$$

for some $c_{1}>0$ and every $t \geq 0$. It follows from [14] that $\left\|A^{\alpha} u(t)\right\|_{2} \leq c_{3}(1+t)^{-\alpha-5 / 4}$ for every $\alpha>0$ and every sufficiently large $t$. If $\mu(t)=c_{4}(1+t)^{-1}$, we get

$$
\begin{aligned}
& c_{3}^{2}(1+t)^{-2 \alpha-5 / 2} c_{1}^{-2}(1+t)^{5 / 2} \geq \frac{\left\|A^{\alpha} u(t)\right\|_{2}^{2}}{\|u(t)\|_{2}^{2}} \\
& \geq c_{4}^{2 \alpha}(1+t)^{-2 \alpha}\left(1-\frac{\left\|E_{\mu(t)} u(t)\right\|_{2}^{2}}{\|u(t)\|_{2}^{2}}\right) .
\end{aligned}
$$

So, if $c_{4}$ is sufficiently large then

$$
1-\frac{\left\|E_{\mu(t)} u(t)\right\|_{2}^{2}}{\|u(t)\|_{2}^{2}} \leq c_{4}^{-2 \alpha} c_{3}^{2} c_{1}^{-2}<1
$$

and

$$
\left\|E_{\mu(t)} u(t)\right\|_{2} \geq c\|u(t)\|_{2}
$$

for every sufficiently large $t$ and some $c>0$.

We will now prove the existence of a constant $c$ such that $\liminf \operatorname{in}_{t \rightarrow \infty}\|u(t)\|_{B_{2, \infty}^{-5 / 2}} \geq c>0$. We proceed by contradiction. Suppose that there exists a sequence $\left\{t_{n}\right\}_{n=1}^{\infty}, \lim _{n \rightarrow \infty} t_{n}=\infty$ such that $\lim _{n \rightarrow \infty} c(n)=0$, where $c(n)=\left\|u\left(t_{n}\right)\right\|_{B_{2, \infty}^{-5 / 2}}=$ $\sup _{j \in Z} 2^{-5 j / 2}\left\|\Delta_{j} u\left(t_{n}\right)\right\|_{2}$. Then

$$
\left\|\Delta_{j} u\left(t_{n}\right)\right\|_{2}^{2} \leq 2^{5 j} c(n)^{2} .
$$

Choose $j_{0}$ so that $2^{j_{0}} \sim\left(1+t_{n}\right)^{-1 / 2}$ and sum up the last inequality over $j$ from $-\infty$ to $j_{0}$. We get

$$
\sum_{j \leq j_{0}}\left\|\Delta_{j} u\left(t_{n}\right)\right\|_{2}^{2} \leq \sum_{j \leq j_{0}} 2^{5 j} c(n)^{2} .
$$

Due to the definition of $\mu,(9)$ and the choice of $j_{0}$, the left hand side is greater than $c\left\|u\left(t_{n}\right)\right\|_{2}^{2}$ for some $c>0$ independent of $n$. The right hand side is smaller than $2 c(n)^{2} 2^{5 j_{0}} \sim 2 c(n)^{2}\left(1+t_{n}\right)^{-5 / 2}$. We get finally

$$
c\left\|u\left(t_{n}\right)\right\|_{2} \leq c(n)\left(1+t_{n}\right)^{-5 / 4}
$$

for every $n \in N$ and this is in contradiction with (8). We sum up the result from this section in the following theorem.

Theorem 5. Let $u_{0} \in B_{2, \infty}^{-5 / 2} \cap L_{\sigma}^{2}$. Let $u$ be a turbulent solution of (1)-(3) with the initial condition $u_{0}$ and such that $\|u(t)\|_{2} \geq c(1+t)^{-5 / 4}$ for some $c>0$ and all $t \geq 0$. Then there exist constants $c^{\prime}$ and $c^{\prime \prime}$ such that

$$
0<c^{\prime} \leq\|u(t)\|_{B_{2, \infty}^{-5 / 2}} \leq c^{\prime \prime}
$$

for every $t \geq 0$.

We will now show that Theorem 5 improves the result presented by Miyakawa in [8]. Miyakawa studied 
turbulent solutions with initial conditions $u_{0} \in L_{\sigma}^{2}$ such that

$$
\int(1+|x|)\left|u_{0}(x)\right| d x<\infty .
$$

He proved that

$$
0<c_{0} \leq\|u(t)\|_{B_{1, \infty}^{-1}} \leq c_{1}
$$

for large $t>0$ and some constants $c_{0}$ and $c_{1}$ if and only if

$$
\begin{array}{r}
\left(\int x_{j} u_{0 m}(x) d x, \int_{0}^{\infty} \int\left(u_{k} u_{l}\right)(x, s) d x d s\right) \\
\neq\left(0, c \delta_{k l}\right) .
\end{array}
$$

Moreover, it was proved by Miyakawa and Schonbek in [9] that (13) holds if and only if there exist constant $c_{0}$ and $c_{1}$ such that

$$
0<c_{0} \leq t^{5 / 4}\|u(t)\|_{2} \leq c_{1}
$$

for large $t>0$.

Since the initial conditions satisfying (11) belong to the space $B_{2, \infty}^{-5 / 2} \cap L_{\sigma}^{2}$, it is clear that Theorem 5 generalizes the result by Miyakawa mentioned above: For initial conditions satisfying (11) and under condition (13) (resp. (14)) both results give lower estimates of $u(t)$, but while Miyakawa's estimate uses the space $B_{1, \infty}^{-1}$, in Theorem 5 we use the space $B_{2, \infty}^{-5 / 2}$. Since $B_{1, \infty}^{-1}$ is continuously embedded into $B_{2, \infty}^{-5 / 2}$, the result from Theorem 5 is stronger. Moreover, Theorem 5 also describes lower estimates for solutions with initial conditions not satisfying (11) (in this paper we have not dealt with their existence).

\section{Appendix}

The definitions and some basic properties of the following concepts can be found in [19]:

- $L^{p}, p \in[1, \infty]$, the Lebesgue space with the norm $\|\cdot\|_{p}$

- $W^{k, p}, k \in N, p \in[1, \infty]$, the Sobolev space with the norm $\|\cdot\|_{k, p}$;

- $C_{0, \sigma}^{\infty}=\left\{\varphi \in\left(C_{0}^{\infty}\right)^{3} ; \nabla \cdot \varphi=0\right\}$, the set of smooth solenoidal vector functions with compact support in $\mathbf{R}^{3}$;

- $L_{\sigma}^{2}$, resp. $W_{0, \sigma}^{1,2}$, the closure of $C_{0, \sigma}^{\infty}$ in $\left(L^{2}\right)^{3}$, resp. $\left(W^{1,2}\right)^{3}$

- $P_{\sigma}$, the orthogonal projection of $L^{2}(\Omega)^{3}$ onto $L_{\sigma}^{2}$;

- $A$, the Stokes operator in $L_{\sigma}^{2}$ defined as $A u=$ $-P_{\sigma} \Delta u$ for every $u \in D(A)=W_{0, \sigma}^{1,2} \cap\left(W^{2,2}\right)^{3}$; $A$ is a positive self-adjoint operator; for the case of the whole space $A u=-\Delta u$;
- $\left\{E_{\lambda} ; \lambda \geq 0\right\}$, the resolution of identity of $A$;

- $A^{\mu}, \mu \in \mathbf{R}$, the powers of $A$ with domains $D\left(A^{\mu}\right)$ and ranges $R\left(A^{\mu}\right)$;

- $\left\{e^{t \Delta} ; t \geq 0\right\}$, the semigroup generated by the Laplace operator $-\Delta$.

Definition 3. If $u_{0} \in L_{\sigma}^{2}$, a measurable function $u$ defined on $\mathbf{R}^{3} \times(0, \infty)$ is called a global weak solution of (1)-(3) if

$$
u \in L^{\infty}\left((0, \infty) ; L_{\sigma}^{2}\right) \cap L^{2}\left((0, T) ; W_{0, \sigma}^{1,2}\right)
$$

for every $T>0$ and the integral relation

$$
\begin{aligned}
\int_{0}^{\infty}\left[-\left(u(t), \partial_{t} \phi(t)\right)+(\nabla u(t), \nabla \phi(t))\right. \\
+(u(t) \cdot \nabla u(t), \phi(t))] d t=\left(u_{0}, \phi(0)\right)
\end{aligned}
$$

holds for all $\phi \in C_{0}^{\infty}\left([0, \infty) ; C_{0, \sigma}^{\infty}\right)$.

Definition 4. A global weak solution $u$ satisfies the strong energy inequality if

$$
\|u(t)\|^{2}+2 \int_{s}^{t}\|\nabla u(\sigma)\|^{2} d \sigma \leq\|u(s)\|^{2}
$$

for $s=0$ and almost all $s>0$, and all $t \geq s$. A global weak solution satisfying the strong energy inequality is called turbulent.

Definition 5. Let $u_{0} \in D(A)$. A function $u \in$ $C([0, \infty) ; D(A)) \cap C^{1}\left((0, \infty) ; L_{\sigma}^{2}\right)$ is called a global strong solution of (1)-(3) if $u(0)=u_{0}$ and $d u / d t+$ $A u+P_{\sigma}(u \cdot \nabla u)=0$ for every $t>0$.

If $u_{0} \in L_{\sigma}^{2}$ then there exists at least one turbulent solution of (1)-(3) (see [4]). Every turbulent solution becomes strong after some transient time (see [19], Chapter V.). This means that there exists $T_{0} \geq 0$ such that $u \in C\left(\left(T_{0}, \infty\right) ; D(A)\right) \cap C^{1}\left(\left(T_{0}, \infty\right) ; L_{\sigma}^{2}\right)$ and $d u / d t+A u+P_{\sigma}(u \cdot \nabla u)=0$ for every $t>T_{0}$.

\section{Conclusion}

In this paper we have presented a survey of some results on the large time decay of energy in turbulent solutions to the Navier-Stokes equations, and the related topic of the large time energy concentration in the frequency space. In the fourth section we improved a result from [8] and showed that some Navier-Stokes flows do not decay asymptotically to zero when considered in suitable Besov spaces.

\section{Acknowledgements}

This work has been supported by the Ministry of Education of the Czech Republic, under project MSM 6840770003. 


\section{References}

[1] Brandolese, L.: Asymptotic bahavior of the energy and pointwise estimates for solutions to the Navier-Stokes equations, Rev. Mat. Iberoamericana, 20, 2004, 223-256.

[2] Bahouri, H., Chemin, J.Y., Danchin, R.: Fourier analysis and nonlinear partial differential equations. [Fundamental Principles of Mathematical Sciences 343], Heidelberg : Springer, 2011.

[3] Chemin, J.Y.: Localization in Fourier space and Navier-Stokes system, Pubbl. Cent. Ric. Mat. Ennio Giorgi, Scuola Norm. Sup., Vol. I, 53, 2004.

[4] Farwig, R., Kozono, H., Sohr, H.: An $L^{q}-$ approach to Stokes and Navier-Stokes equations in general domains, Acta Math., 195, 2005, 21-53.

[5] Kajikiya, R., Miyakawa, T.: On $L^{2}$ decay of weak solutions of the Navier-Stokes equations in $R^{n}$, Math. Z., 192, 1986, 135-148.

[6] Kato, T.: Strong $L^{p}-$ solutions of the NavierStokes equations in $\mathbf{R}^{m}$, with applications to weak solutions, Math. Z. 187 1984, 1471-480.

[7] Leray, J.: Sur le mouvement d'un liquide visqueux emplissant l'éspace, Acta Math. 63, 1934, 193-248.

[8] Miyakawa, T.: On upper and lower bounds of rates of decay for nonstationary Navier-Stokes flows in the whole space, Hiroshima Math. J., 126, 2002, 431-462.

[9] Miyakawa, T., Schonbek, M.: On optimal decay rates for weak solutions to the Navier-Stokes equations in $\mathbf{R}^{n}$, Math. Bohem., 126, 2001, 443455.

[10] Scarpellini, B.: Solutions of evolution equations of slow exponential decay, Analysis 20 2000, 255283.

[11] Schonbek, M.: Asymptotic behavior of solutions to the three-dimensional Navier-Stokes equations, Indiana University Mathematics Journal, 41, 1992, 809-823.

[12] Schonbek, M.: Large time behavior of solutions to the Navier-Stokes equations, Comm. Partial Differential Equations, 11 1986, 753-763.

[13] Schonbek, M.: Lower bounds of rates of decay for solutions to the Navier-Stokes equations, Journal of the American Mathematical Society, 4, 1991, 423-449.
[14] Schonbek, M., Wiegner, M.: On the decay of higher-order norms of the solutions of NavierStokes equations, Proceedings of the Royal Society of Edinburgh, 126A, 1996, 677-685.

[15] Skalák, Z.: Some aspects of the asymptotic dynamics of solutions of the homogeneous NavierStokes equations in general domains, J. Math. Fluid Mech., 12, 2010, 503-535.

[16] Skalák, Z.: On the asymptotic decay of higherorder norms of the solutions to the Navier-Stokes equations in $\mathbf{R}^{3}$, Discrete Contin. Dyn. Syst. Ser. $S, \mathbf{3}, 2010,361-370$.

[17] Skalák, Z.: Large time behavior of energy in exponentially decreasing solutions of the NavierStokes equations, Nonlinear Analysis, 71, 2009, 593-603.

[18] Skalák, Z.: Solutions to the Navier-Stokes equations with large time energy concentration in low frequencies, ZAMM, 91, 2011, 733-742.

[19] Sohr, H.: The Navier-Stokes Equations, An Elementary Functional Analytic Approach. Basel, Boston, Berlin : Birkhäuser Verlag, 2001.

[20] Wiegner, M.: Decay results for weak solutions of the Navier-Stokes equations on $\mathbf{R}^{n}$, J. London Math. Soc., 35, 1987, 303-313. 